\title{
Energy-aware Routing to a Mobile Gateway in Wireless Sensor Networks
}

\author{
Kemal Akkaya and Mohamed Younis \\ Dept. of Computer Science and Electrical Engineering \\ University of Maryland Baltimore County \\ Baltimore, MD 21250 \\ $\{$ kemal1,younis $\} @$ cs.umbc.edu
}

\begin{abstract}
Almost all of the energy-aware routing protocols that have been proposed for wireless sensor networks aim at optimizing network performance while relaying data to a stationary gateway (sink). However, mobility of the gateway can make routes established through such contemporary protocols, unstable and non-optimal. The use of mobile gateways introduces a non-traditional trade-off between the need for frequent re-routing to ensure optimal network operation and the desire for minimizing the overhead of topology management. In this paper, we propose an energy aware mechanism for efficient and continual data delivery to a moving gateway. Our approach tracks the distance of the gateway from the last hops to the gateway, and dynamically adjust the routes either via increasing the last hop nodes' radio ranges or introducing new forwarder nodes as part of the routes. Rerouting is triggered when the current routes become unacceptably inefficient. The presented approach is validated in a simulation environment.
\end{abstract}

\section{INTRODUCTION}

Networking unattended wireless sensors is expected to have significant impact on the efficiency of many civil applications, such as disaster management, environment monitoring and security [1]. These systems gather data from multiple sensors and process these data at a gateway node in order to monitor events in an area of interest. Sensor nodes are typically battery-operated and in many applications battery replacement or recharging is impossible or impractical. Such energy constraints combined with a typical deployment of large number of sensor nodes have necessitated energy-awareness at most layers of networking protocol stack including the network layer. Efficient routing of sensor data has received significant attention from the technical community. Current research on routing in wireless sensor networks focuses on stationary sensor and gateway nodes and mostly aims at maximizing the lifetime of the network, allowing scalability for large number of sensor nodes and supporting tolerance for sensor damage and battery exhaustion [2].

However, in numerous applications the mobility of the gateway is desirable. One of the possible configurations of a mobile gateway can be a computer installed on a moving object. Examples of this configuration include an emergency vehicle equipped with computing and communication devices in the context of a disaster management application and a jeep with a laptop computer in a battle environment. The gateway in such setup changes its location and thus the network topology will be modified so frequently that the increased control message traffic can diminish the energy savings obtained by setting multi-hop paths. On the other hand, being irresponsive to changes in the gateway location can lead to high packet losses and negatively affect the application of the sensor network. Therefore, the gateway mobility requires careful handling to limit the overhead of excessive topology management, maintain low packet loss ratio and still thrive to achieve efficient network operation.

In this paper, we present a new mechanism for efficient routing of data traffic to a mobile gateway in wireless sensor networks where multiple active source sensors are generating data simultaneously at different parts of the sensed region. The proposed approach assumes that energy efficient multihop routes can be set at the initial gateway location by using any distributed or centralized sensor routing protocol. Those routes are used to relay the data as long as the gateway is within the transmission range of last hop nodes i.e., sensors that are directly transmitting to the gateway. When the gateway goes out of range of last hop sensors, the current routes are extended by the involvement of new nodes which we call forwarders. The forwarder nodes relay data from the last hop nodes so that continual delivery of messages is maintained when the gateway is on the move. Rerouting based on the new position of the gateway is performed only when the current route setup becomes fairly inefficient to provide the desired network operation. Minimizing the number of rerouting operations will help in reducing the network management overhead and thus the energy usage. This paper explores the non-traditional tradeoff that exists between the desire for a frequent re-routing to handle gateway mobility and the minimization of the network management overhead due to frequent rerouting. To the best of our knowledge, such tradeoff has not been investigated in the context of sensor networks before. In addition, we introduce a new mobility model for the movement of the gateway that will best fit applications of sensor networks in a battle environment.

In the balance of this section, we describe the system model and survey the related work. Section 2 presents our approach for efficiently handling the gateway mobility in terms of energy usage. Section 3 is dedicated to the experimental validation, describing the simulation environment and analyzing performance results. Finally, we conclude the paper in section 4 . 


\subsection{System Model}

A set of sensors is spread throughout an area of interest to monitor particular events. The sensors are battery-operated and are empowered with limited data processing engines. Sensors are identifiable by their geographical location and functional capabilities. The mission for these sensors is dynamically changing to serve the need of an application. A gateway node, which is significantly less energy-constrained than the sensors, is deployed in the physical proximity of sensors. Sensors are assumed to be within the communication range of the gateway node. The gateway is responsible for organizing the activities at sensor nodes to achieve a mission and fusing data collected by sensor nodes. While sensor nodes are stationary, we are considering a model for a gateway with full mobility.

The sensing, processing and radio circuits of a sensor can be independently turned on and off. In addition, it is assumed that the radio transmission power can be programmed for a required range up to a known maximum. It is also assumed that the sensor can act as a relay to forward data from another sensor. It is worth noting that most of these capabilities are available on advanced sensors, e.g. the SenTech Acoustic Ballistic Module [3].

\subsection{Related Work}

Many routing protocols (e.g. AODV [4], DSR [5]) have been proposed for mobile ad hoc networks in order to efficiently deal with the mobility of the nodes. However, such protocols cannot be directly applied to our case where the gateway is mobile. This is due to several reasons: First, sensor nodes are deployed in sheer numbers and therefore there is no global addressing scheme such as IP numbers to be used as in mobile ad hoc networks. Second, their energy and resources are very scarce and has to be managed wisely. Therefore, using many control packets to build the routes is undesirable. Most of the mentioned routing protocols for mobile networks are based on exchanging many control packets before setting the routes. Third, the data gathering model in sensor networks is from many sources to one sink which is not the case in mobile ad hoc networks.

Very little research has addressed possible mobility of the sink (gateway) node in wireless sensor networks. A protocol, called TTDD, to consider such mobile sinks is described in [6]. The protocol initially builds a grid structure which divides the network into cells with several dissemination nodes. The dissemination nodes are responsible for relaying the query and data to/from the proper sources. When data is needed by a sink, the queries are flooded locally within the cell until it reaches a dissemination node. A data path from the source to the dissemination node is then established. TTDD can work well with event-driven systems where sources are queried on demand, but it will not be suitable for applications where sources generate burst data. In such circumstances, the entire path would have to change when the sink switches to another dissemination node, a case that can happen too often for small cells. Large cells on the other hand increase the energy consumption due to the local flooding.

SEAD is another mechanism for routing sensor's data to mobile sinks [7]. The idea is to construct a minimum Steiner tree for the mobile sinks and designate some nodes on the tree as access points. Each mobile sink register itself with the closest access node. When the sink moves out of range of the access node, the route is extended through the inclusion of a new access node. Such partial path extension is allowed only for a limited number of hops and then the branch of the Steiner tree for that sink is modified by finding a least cost path to the sink. Our work differs from SEAD in three aspects. First, we consider multiple active sources that generate data simultaneously, a case that SEAD would not handle. Second our algorithm can be easily integrated with both centralized and distribute routing approaches. Third, we evaluate different mobility patterns for the sink node that would fit best to the requirements of applications such as target tracking, disaster management, etc.

\section{ROUTING TO A MOBILE GATEWAY}

In this section we define the problem and analyze the overhead and tradeoffs related to the handling of gateway mobility and describe an efficient algorithmfor dealing with such mobility.

\subsection{Problem Definition}

In application setups where the gateway moves, ensuring uninterruptible data traffic to the gateway is challenging. The main problem with a mobile gateway is the issue of dynamic maintenance of multihop routes in order to provide same desired service as if the gateway is stationary. This requires frequent acknowledgment of the gateway's location to the network causing significant overhead in terms of energy and bandwidth of sensor nodes. Therefore, we need a mechanism that will not only handle the mobility efficiently but also try to maximize the lifetime of the network. Based on these observations we define the problem as follows:

"Given a gateway moving with a certain mobility model and established initial routes for delivery of sensor data, we are interested in providing uninterrupted data delivery to the gateway by minimally affecting the network topology and at same time increase the lifetime of the network".

\subsection{Design Issues}

Gateway mobility introduces some issues that can impact the performance of the network. In this subsection, we highlight these issues.

Functionality-efficiency tradeoff: An intuitive approach for handling the gateway motion is to continually readjust the network topology based on the new locations of the gateway. Such rerouting needs to be done very frequently and new routes should be broadcasted to the network in order to avoid the loss of data packets. A second approach is to embed redundant routes in the initial setup anticipating the gateway 
travel path so that data continues to be received by the gateway. Such approach can be very inefficient in terms of energy consumption with a worst-case performance that is equivalent to network flooding, and thus this approach would be undesirable for sensor networks. A third possibility is for the sensor nodes at the end of each path to adjust their transmission range based on the future location of gateway as long as it is feasible to reach such location. Once the gateway becomes out of range, the current routes can be extended using the last-node on a path as a source and designating new nodes to relay the data to the gateway.

A tradeoff does exist between these three approaches. Given the inefficiency of the second alternative, we focus on the first and the third approaches. While ensuring gateway reachability to last hops through increasing the transmission power and designating ad hoc forwarders can quickly make the network operation inefficient and increase the latency by introducing extra hops to be traveled, frequent changes to the route setup, can raise the overhead of topology maintenance to an unacceptable level as explained below. Balancing the need for frequent re-routing to ensure network functionality and the desire for minimizing the overhead of network management has not been addressed before in the literature. Our algorithm, as described later, exploits this tradeoff.

Rerouting overhead: Establishing a new network topology can introduce significant overhead in terms of energy consumption. When distributed protocols are used for route set up, data delivery is disrupted to allow for a typically large flow of control packets [1][2]. Frequent rerouting can cause thrashing with nodes consuming more time and resources on control traffic than data. On the other hand, if the gateway sets up the routes, it has to broadcast these routes in order to inform the sensor nodes about their role. Since a number of management strategies for sensor networks, e.g. [8][9], turn idle sensors to low-power sleep mode to save their energy, such broadcast requires either all sensor nodes to be continuously active or nodes in the sleep mode to switch to an active state on-time for hearing such route update. Keeping sensors active all the time can deny the network an opportunity for energy conservation. In addition, excessive state transition from active to sleep mode can also be a significant energy burden [10]. The network will even more burdened in case the routing protocol requires sensors to acknowledge the receipt of routing messages and to engage with other nodes in establishing connections.

\subsection{Proposed Approach}

We pursue an approach based on the current network state. The gateway is assumed to move in strides to reach intermediate positions. The strides are to be in straight line. This is similar to piece-wise linear approximation of curves in analytical geometry. The size of the strides can be determined based on the gateway travel speed. It should be noted that the gateway speed is assumed to be reasonable enough to handle. We will capture the effect of gateway

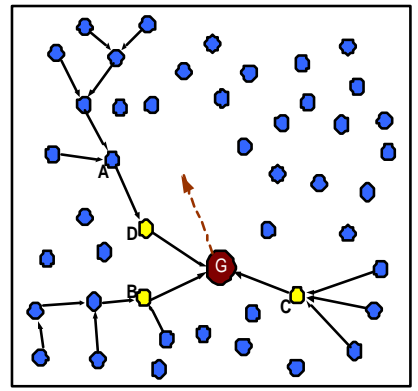

(i)

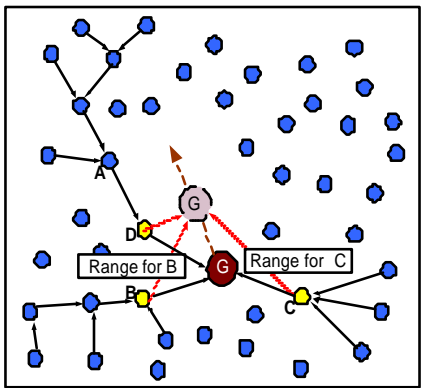

(ii)
Fig. 1 : (i) Topology at start of a stride (ii) $\mathrm{B}$ and C adjust their transmission range while $\mathrm{G}$ is on the move.

speed on performance experimentally in section 3. Energy efficient routes are set at the initial gateway location so that the gateway keeps receiving the messages uninterruptedly till the next intermediate position. Note that any routing algorithm for sensor networks either centralized (e.g. LEACH [11]) or distributed (e.g. Directed Diffusion [12]) can be used in order to set the initial multi-hop paths.

Given the energy and delay tradeoff associated with the frequency of rerouting, we pursue a mechanism that balances the interest in maintaining efficient network operation and avoiding excessive rerouting overhead. To limit the frequency of rerouting, our gateway's motion handling mechanism tries to maintain continual packet delivery to the gateway through designating some forwarders to extend the current routes. A new network topology is formed when the current routes become either inefficient in terms of energy consumption or the number of forwarders for a last hop node exceeds a given threshold. When the gateway starts to move in strides, the network topology is reassessed at each gateway's intermediate position (end of a stride). The reassessment concludes in one of three options: (1) The gateway can still be reachable by the last hops while traveling on the next stride, (2) the path should be extended (3) rerouting is necessary.

If the gateway still lays within the reachable range of the last hop nodes on the current routes, even at the end of the stride, the gateway will simply instruct these nodes to adjust the transmis sion power of their radio to cover the gateway's next move. Such situation is shown in Fig. 1(i)-(ii). The ranges of last hop nodes $\mathrm{B}$ and $\mathrm{C}$ are increased to maintain connectivity to the gateway. While increasing the transmission power defies the energy efficiency achieved through the pursuit of multi-hop routing, the impact of such process on energy consumption will be limited in scope since only few nodes are affected. In addition, some of the last hop nodes such as node D can save energy since it will be become closer to the gateway.

If the gateway detects that it will go out of the maximum transmission range of some of the last hop nodes, the second option of extending the data paths is to be explored. In this case, a sensor node is solicited to forward the packets from the last hop of what would be a discontinued path. Based on the routing methodology the forwarder can be identified by the 


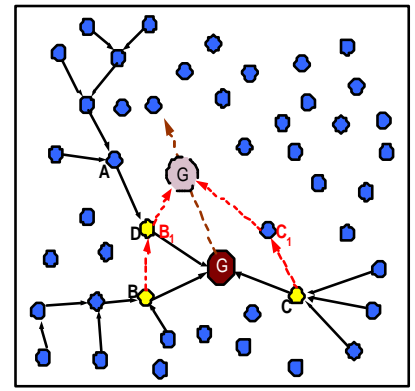

(i)

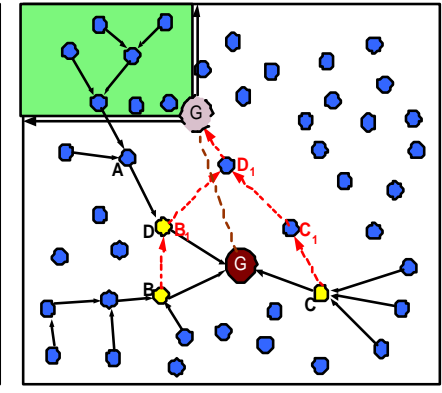

(ii)
Fig. 2: (i) $B$ and $C$ find new forwarders, i.e. $B_{1}$ and $C_{1}$ to relay their data when the gateway is out of their transmission range, (ii) When the number of forwarder for $C$ exceeds $1\left(C_{1}\right.$ and $\left.D_{1}\right)$, the gateway starts reassessment on the efficiency of the routes in the dotted region to decide on the urgency for rerouting.

gateway or by the last node on the path. In case of a centralized route setup, the gateway picks a suitable node based on its proximity to the gateway's next intermediate position and extends the data paths by notifying the last node on the path. For distributed data routing the node at the end of the path will pick an appropriate forwarder among its neighbors and get the picked forwarder engaged in data relaying. A pictorial illustration of this situation is shown in Fig. 2(i). When the gateway gets out of the reachable range of B and C, forwarders would be needed in order to relay B's and $C$ 's data. Nodes $B_{1}$ and $C_{1}$ are selected as forwarders and the routes are updated as seen in the figure. $B_{1}$ and $C_{1}$ act as relay nodes as long as the gateway is in their transmission range. While $C_{1}$ is an example for the unused forwarder, $B_{1}$ has to accommodate the additional traffic. New forwarders are found when gateway goes out of transmission range of $B_{1}$ and $C_{1}$ and such process continues until the next rerouting. Among multiple potential forwarders, the closest node to the next gateway intermediate location is favored since that would increase the probability that the node will not need an additional forwarder on the next travel stride. If a forwarder node cannot be found, rerouting becomes a necessity.

It should be noted that extension of routes through designating forwarder nodes can quickly make the network topology inefficient. Therefore, we impose a certain bound on the number of forwarders that can be augmented to a particular path. When such bound is reached, our algorithm starts checking the efficiency of the current routes in order to qualify the need for new routes. Since rerouting can be costly in terms of energy consumption as explained before, our approach prefers to keep the current route setup unless a major inefficiency in terms of energy is detected. This is done by checking the routes from source sensors within the direction of the gateway motion. The algorithm assumes the gateway's current coordinates to be $(0,0)$ and considers the quarter that it is moving into, as seen in Fig. 2(ii). For application with large number of active sources, the scope of consideration can be limited to those located not very far from the current location of the gateway. For example, in Fig 2(ii) data routes from sources in the shaded area most probably become inefficient when the gateway is approaching these sources. After the selection of sources, the gateway makes a comparison as shown below:

$$
\sum_{i \in S}\left(P R(i)^{*}\left(\sum_{l \in E P(i)} T E(l)\right)\right)-\sum_{i \in S}\left(P R(i) *\left(\sum_{l \in C P(i)} T E(l)\right)\right)>\delta
$$

where,

$\mathrm{S}$ : Set of source nodes in the considered region

PR(i) : Packet generation rate of node $i$

TE(1) : Transmission energy cost for a packet on link $l$

$\mathrm{EP}(\mathrm{i})$ : Estimated shortest path from source node $i$ to gateway

$\mathrm{CP}(\mathrm{i})$ : $\quad$ Current path from source node $i$ to gateway

$\delta$ : The threshold value for triggering a new reroute

The gateway basically uses the total energy for packet transmission to check whether the current routes from these sources are too inefficient. The gateway estimates what would be the least energy path from these sources in order to qualify the degree of efficiency of current paths. If there is a significant difference, i.e. exceeding a threshold, the gateway pursues the third option of establishing a new topology. Otherwise, the algorithm continues to designate forwarder nodes. If the gateway does not know the exact locations of all deployed sensors, it can consider nodes that are currently part of a path or have been relaying data before. A direct link from the source to the gateway can be used in the energy efficiency assessment when the gateway is unaware of the potential nodes that can relay the data from the source.

\subsection{Pseudo Code and Complexity Analysis}

The proposed algorithm is shown in Fig. 3. At each stride, the algorithm checks each sensor in the list of nodes that are one hop away from the gateway to check whether the gateway $(\mathrm{G})$ is to get out of the range of that sensor or not (line 2-3). If the gateway is still in range, it estimates the next intermediate position and sets the transmission range for the last hop node so that the gateway receives the messages uninterruptedly until reaching that intermediate position (line 8). If the gateway will be out of range, a new forwarder is found for that particular node in line 7. Each time a new forwarder is to be found, the number of forwarder nodes for that path is checked in line 4. If that number is bigger than a given bound (e.g. $k$ ), the algorithm makes an assessment to find out whether new routes are needed or not in lines 5-6. If the gateway is unaware of the sensor state, it can use a constant maximum range for all sensors.

MakeAssessment function initially identifies the source nodes to be checked in lines 1-3. The idea is to assume the coordinates of $\mathrm{G}$ to be $(0,0)$ and normalize the coordinates of all sensors with respect to G's location. After such normalization, the source nodes are easily identified by looking at the movement direction of G. In lines 4-5, the shortest paths from source nodes to $G$ are found. Then, the total transmission cost for both shortest routes and current routes are computed in lines 6-7. Based on the comparison in line 8 , a reroute is requested if necessary in line 9. 
Line 2 of FindForwarder function reflects the criteria for selecting the most appropriate node among multiple potential forwarders. While the closeness to the gateway is the criterion used in the algorithm, other factors such as remaining energy, if known, can be easily incorporated. If a forwarder in not found, a new topology is requested (line 5).

To assess the complexity of the relocation algorithm, we consider each function separately. The complexity of MakeAssessment function mainly depends on the number of nodes in the region that the gateway moves to. Let the number of such nodes to be $s$ and the number of links between those nodes to be $q$. Then the complexity of finding the shortest paths for those nodes will be $O(q * \log (s))$ assuming the use of Dijkstra's shortest-path algorithm The

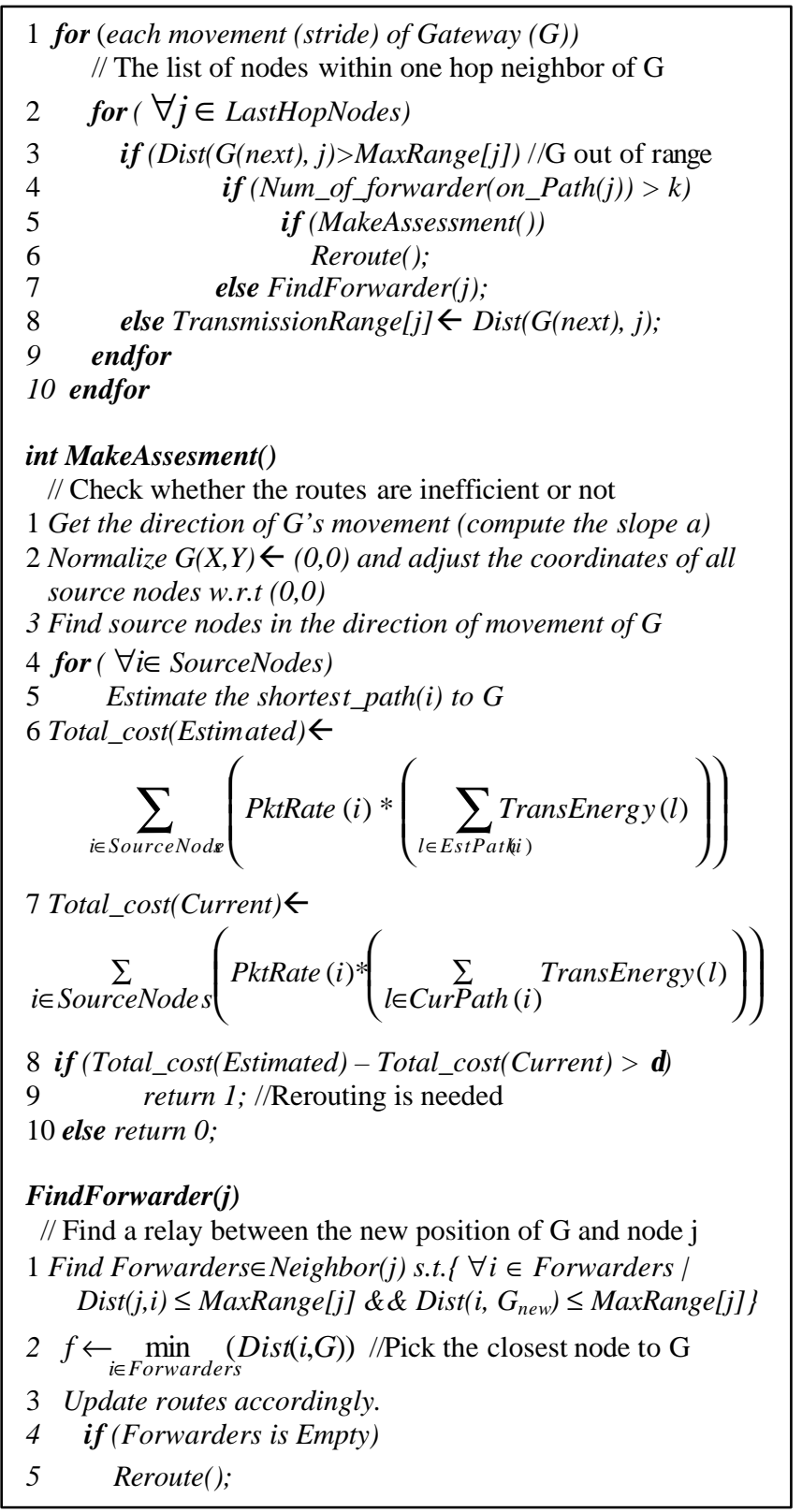

Fig. 3: Pseudo code for handling gateway motion assessment operation is only done for the paths which exceeded the bound for the number of forwarder nodes. Let such number of paths be $k$, the number of nodes in LastHopNodes be $l$ and the total number of sensor nodes in the network be $n$. Note that $k$ and $l$ are typically much less than $n$ $(k<l<n)$. The overall complexity of MakeAssessment will be $k * q * \log (s)$ which will be $O\left(n^{2} \log (n)\right)$ in the worst case.

For the FindForwarder function, the number of iterations to skim through the list LastHopNodes will be $O(l)$. When the gateway is moved to a new location and loses connection with some of the sensors in LastHopNodes, a forwarder is to be found for each of such sensors. In order to find a forwarder for a particular node $i$, we need to skim through its neighbor list, Neighbor(i). In the worst case, $\sum_{i=1}^{l} N e i g h b o r(i)$ iteration is made since in the worst case, a forwarder needs to be identified for every node in the LastHopNodes list and all neighbor nodes of a particular sensor may be a potential forwarder. Therefore, the total number of iterations will be in the order of $O(l)$ which is $O(n)$ in the worst case.

We argue that such complexity would not make our handling of the gateway motion a major resource burden.

\section{EXPERIMENTAL VALIDATION}

The effectiveness of our approach for handling gateway mobility is validated through simulation. This section describes the underlying network operation, simulation environment, performance metrics and experimental results.

\subsection{Validation Environment}

We have adapted the system architecture of [12] for validating our approach. The gateway electively engages some sensors in probing the surroundings based on missions that are assigned to the network. Unselected sensors can switch to a low-energy sleep mode. A TDMA-based MAC protocol is used allowing nodes to switch to sleep mode when they are not communicating. Rerouting is triggered by either an application-related event that requires the involvement of different set of sensors, a need for a more efficient network topology to suit the gateway movement or the depletion of the battery of an active node. The gateway sets up data paths using the minimum distance squares routing methodology.

Experiment Setup: In the experiments, the network consists of 50 to 200 randomly placed sensors deployed in a $500 \times 500$ $\mathrm{m}^{2}$ area. The gateway initial position is determined randomly within the area boundaries. A free space propagation channel model is assumed [14] with the capacity set to $2 \mathrm{Mbps}$. The size of a data packet is $10 \mathrm{Kbit}$ [3]. Each node is assumed to have an initial energy of 5 joules. A node is considered nonfunctional if its energy level reaches 0 . The transmission range for a sensor node is assumed to be $50 \mathrm{~m}$ [15]. For a node that is actively sensing the environment, packets are generated at a constant rate of 1 packet/sec. This value is consistent with the specifications of the SenTech Acoustic Ballistic Module [3]. 
Each data packet is time-stamped when it is generated to allow the calculation of average delay per packet.

In the experiment we assume that the network is tasked with a target-tracking mission. The initial set of sensing nodes is chosen to be the nodes on the convex hull of sensors in the deployment area. The set of sensing nodes changes as the target moves. Targets are assumed to come from outside the area and to move in straight lines. Each target has a constant speed chosen uniformly in the range 4-6 meters/s.

Mobility Models: We have considered two different mobility models for the gateway motion. The first is the popular Random Waypoint model [16], which is widely used in simulating mobile ad hoc networks. The other one is a new model that we propose for battle environments where the motion of the gateway is dynamically restrained in order to escape possible enemy or target locations. We call this new model the Target Avoidance Model. The gateway selects the destinations based on the current traffic information where high volume traffic in a location refers to a possible detection of a target. The gateway will strive to find a location that is equidistant and symmetric from the possible targets or enemy locations in the battle field. We have assumed at most two targets to be active in the deployment area. A new target is generated when either one or two of the current targets leave the area. An example is shown in Fig. 4. The gateway first identifies symmetric points $\mathrm{A}$ and $\mathrm{B}$, with respect to its position, for the estimated current locations of targets $T_{1}$ and $\mathrm{T}_{2}$. The destination for the gateway can be selected randomly on or beyond the line $\mathrm{AB}$ through the north-east of the region to avoid $T_{1}$ and $T_{2}$. In this model, the determination of the speed of the gateway towards a destination $\left(S\left(t_{i+1}\right)\right)$ will be based on its speed for reaching the previous destination, i.e., $\mathrm{S}\left(\mathrm{t}_{\mathrm{i}+1}\right)=\mathrm{S}\left(\mathrm{t}_{\mathrm{i}}\right) \pm \operatorname{random}(1, \mathrm{X})$ where $\mathrm{X}$ can be selected the maximum speed increment. The random value is added/subtracted $50 \%$ of the time. This is different than Random Waypoint model where speed is selected randomly between 1 and a maximum value.

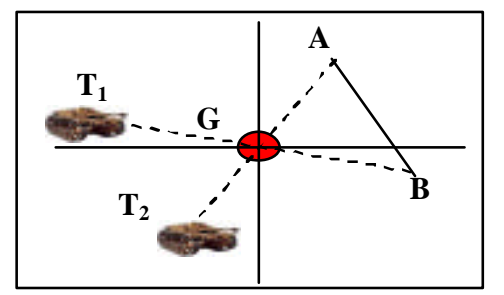

Fig. 4: The gateway picks a position on $\mathrm{A} \rightarrow \mathrm{B}$ to avoid targets

Performance Metrics: Given our interest in minimizing the impact of gateway mobility on network performance, we used the following metrics:

- Network throughput: Defined as total number of packets received by the gateway divided by the simulation time.

- Average delay per packet: Defined as the total time for a packet to reach the gateway from the source node. This metric gives an idea of how efficient the multihop routes are in terms of hop count.
- Average lifetime of a node: This metric gives a good measure of the network lifetime and show how efficient is the algorithm in energy consumption.

\subsection{Performance Results}

In this section, we present some of the obtained performance results. The performance of our approach is captured under the two mentioned mobility models. We named our approach as EARM (Energy-Aware Routing to Mobile Gateway) and compare the measured performance to that of a baseline approach in which the transmission power of last hop nodes is adjusted as long as the gateway stays within their range and rerouting is performed when the gateway becomes unreachable to them. We have used the random waypoint model (RWP) and target avoidance model (TA) for the motion of the gateway. We have applied 5 distinct seeds in order to generate random network topologies. Separate simulation experiments were performed for each topology. Each simulation lasted $2000 \mathrm{sec}$. We observed that with $90 \%$ confidence level, the simulation results stay within $6 \%-10 \%$ of the sample mean.

Rerouting overhead: Frequent adjustment of the network topology when the gateway goes out of range of sensor nodes, will introduce significant overhead in terms of energy consumption as explained in section 2.2. In our simulation network setup such overhead is due to increased number of state changes from active to sleep mode and to extended active time. Therefore, we conducted experiments in order to observe the effect of rerouting frequency on energy consumption. We have varied the reroute period i.e. the number of data collection cycles to pass before the next reroute. We have also studied how such effect relates to the number of sensors. The results, depicted in Fig. 5, have shown that the total transition energy due to state changes increases significantly with a smaller reroute period. Such effect is very much expected. Moreover, we observe from Fig. 6 that when the number of sensors increases significantly, the overhead of rerouting becomes a big portion of the total communication energy. Therefore, frequent rerouting can be undesirable especially when the network size is big.

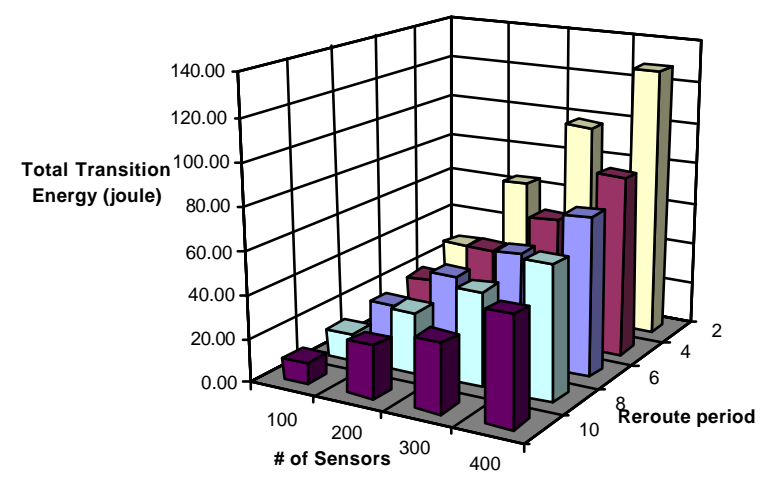

Fig. 5: Total overhead-related energy with respect to different number of sensors and varying reroute periods. 


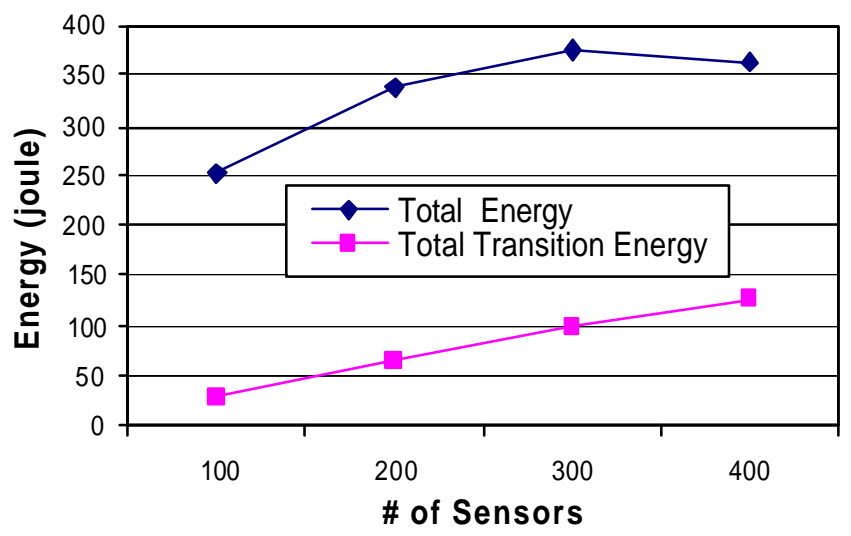

Fig. 6: Total energy and state transition energy for increased number of sensors.

Performance under different network size: In this set of performance experiments, we have varied the number of sensors and fixed the gateway speed at $10 \mathrm{~m} / \mathrm{sec}$. When considering the network throughput (Fig. 7), our approach under random waypoint model (EARM-RWP) performs close to the baseline-RWP especially for small network sizes. The slight difference is due to the fact that the baseline approach frequently obtains the most energy-efficient and shortest routes by adjusting the network topology. Short transmission range reduces the error rate, suggesting a positive impact on the network throughput. Similar observations are true for Target Avoidance (TA) Model.

We have also observed that our approach performs better under the random waypoint model (EARM-RWP) than the target avoidance model (EARM-TA). This is because the gateway in the target avoidance model moves away from data sources and hence extends the multi-hop paths. The increase in distance from the sources and in number or hops boosts the possibility of packet drops and significantly lowers the throughput. The increase in the number of hops also causes the end-to-end delay to increase in comparison to the baseline approach under both mobility models as confirmed in Fig. 8 .

While frequent rerouting enhances the throughput and the end-to-end delay, the overhead eats up most of the sensor's energy lowering the average lifetime of a node, as seen in Fig. 9. Our approach provides at least $20 \%$ increase in node lifetime for both used mobility models and this enhancement grows significantly with the increasing network size. It should be noted that in other setups the overhead due to rerouting can be even more damaging [12].

Experimenting with different gateway speed: We have also studied the effect of the gateway travel speed on performance. The results in Fig. 10 and 11 show that our algorithm EARM-RWP and EARM-TA performs very close to the baseline-RWP and baseline-TA respectively, demonstrating the ability of our algorithm to efficiently handle gateway motion. Again the performance under the random waypoint model is better than that of the target avoidance model since the latter causes the gateway to be far from the source of data traffic.

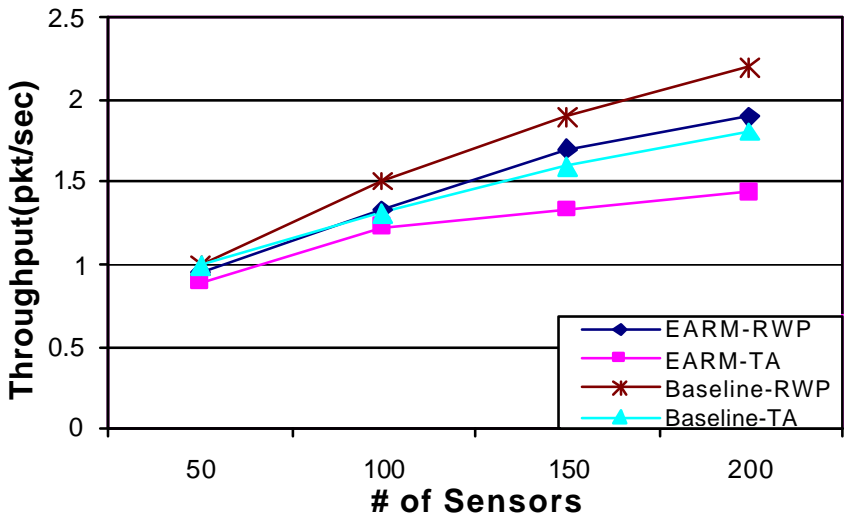

Fig. 7: Network throughput with varying number of sensors.

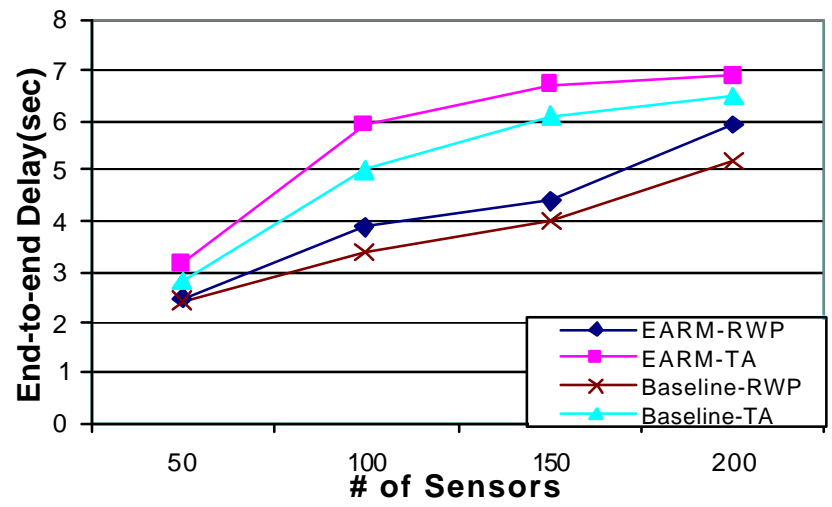

Fig. 8: End-to-end delay with varying number of sensors.

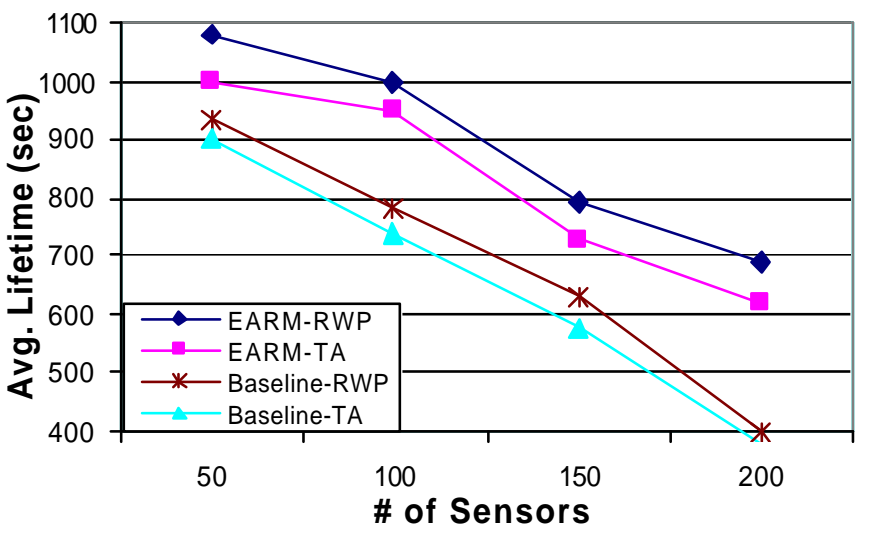

Fig. 9: Average lifetime of a node with varying number of sensors.

Fig. 12 again confirms how our approach prevents the fast gateway's motion from triggering excessive topology adjustment that shortens the network lifetime.

\section{CONCLUSION}

In this paper, we have pres ented an efficient approach for routing sensors data to a mobile gateway. The presented approach conducts a trade-off between the frequency of network topology adjustment to maintain efficient route setup and the overhead that such adjustment introduces to the 
sensors. The basic idea of our approach is to track the gateway's proximity to the last nodes on current data paths and selectively adjusts the transmission power of some of these nodes to ensure continual packet delivery to the gateway. When the gateway gets out of range, new forwarder nodes are identified and appended to the existing routes. When the number of forwarders on a particular route exceeds a pre-defined bound, the impact of rerouting is qualified and the network topology is updated if the overhead is justified.

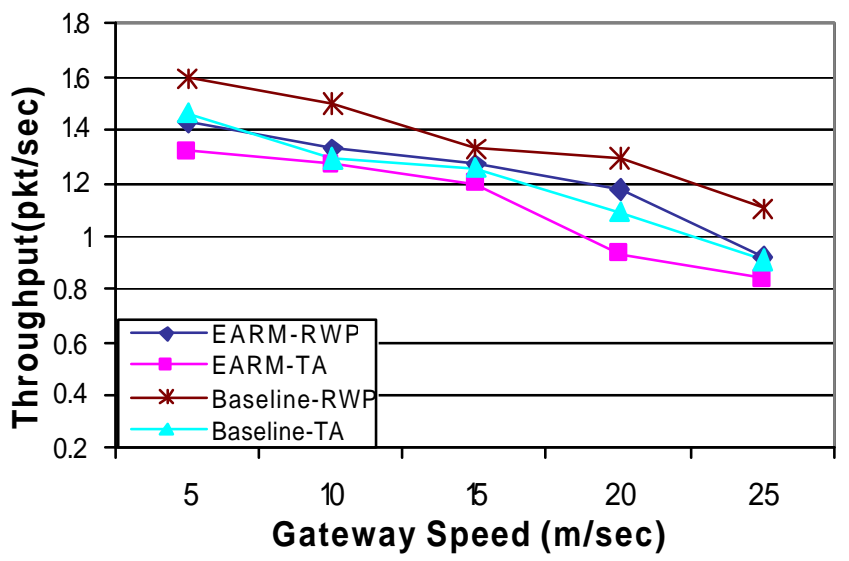

Fig. 10: Network throughput for varying gateway speed.

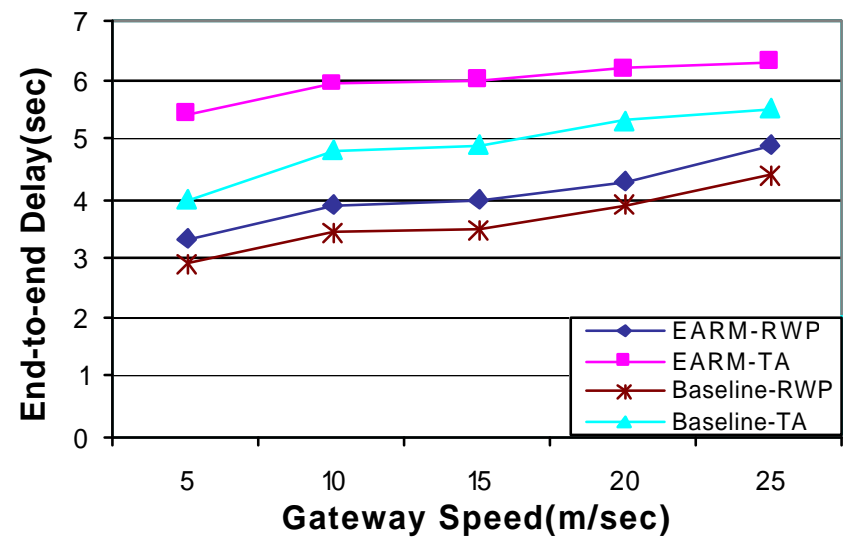

Fig. 11: End-to-end delay for varying gateway speed.

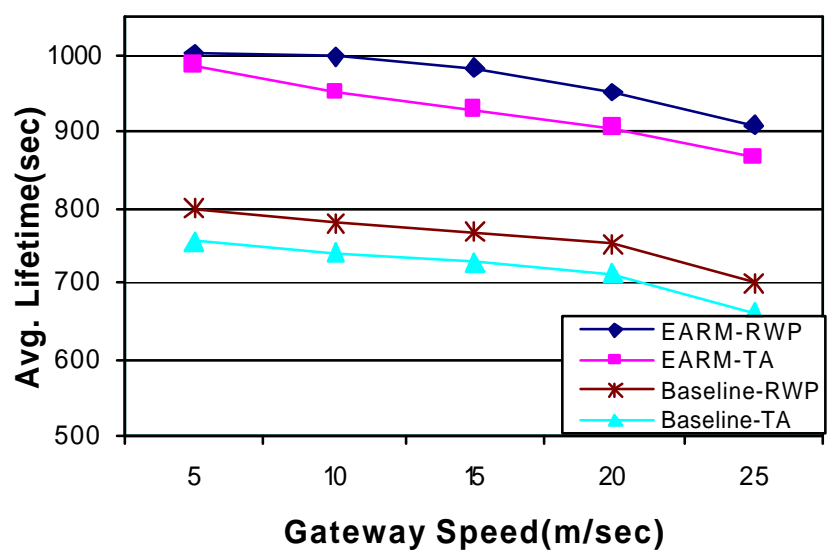

Fig. 12: Average lifetime of a node for varying gateway speed.
The performance of our approach is studied through simulation. In addition to the popular random waypoint model, we have introduced a new mobility model, namely Target Avoidance Model, for applications of sensor networks in battle environments. Simulation results have shown that our approach handles the motion of the gateway efficiently providing at least $20 \%$ longer lifetime for the network at the cost of a slight increase in end-to-end delay in comparison to the performance achieved through continual rerouting.

\section{REFERENCES}

[1] I. F. Akyildiz et al., "Wireless sensor networks: a survey", Computer Networks, Vol. 38, pp. 393-422, 2002.

[2] K. Akkaya and M. Younis, "A Survey on routing protocols for wireless sensor networks", Ad Hoc Networks (in press).

[3] "Data sheet for the Acoustic Ballistic Module", SenTech Inc., http://www.sentech-acoustic.com/

[4] C. E. Perkins and E. M. Royer , "Ad-hoc On Demand Distance Vector Routing," Proceedings of WMCSA'99, February 1999.

[5] D. B. Johnson and D. A. Maltz, "Dynamic Source Routing in Ad Hoc Wireless Networks," in Mobile Computing, Chapter 5, pages 153-181, Kluwer Academic Publishers, 1996.

[6] H. Luo et al., "TTDD: Two-tier Data Dissemination in Largescale Wireless Sensor Networks," ACM/Kluwer Mobile Networks and Applications (MONET), to appear.

[7] H. Kim, T. Abdelzaher, and W. Kwon, "Minimum-energy asynchronous dissemination to mobile sinks in wireless sensor networks", Proceedings of the $1^{\text {st }}$ international conference on Embedded networked sensor systems, Los Angeles, CA, 2003.

[8] P. Havinga and G. Smit, "Energy-efficient TDMA medium access control protocol scheduling," Proc. of the Asian Intl. Mobile Computing Conference (AMOC 2000), November 2000.

[9] K. Arisha et al., "Energy -Aware TDMA-Based MAC for Sensor Networks," Proc. of the IEEE Workshop on Integrated Management of Power Aware Communications, Computing and Networking (IMPACCT 2002), New York, May 2002.

[10] E. Shih et al., "Physical layer driven protocol and algorithm design for energy -efficient wireless sensor networks," Proc. of $7^{\text {th }}$ Intl. Conf. on Mobile Computing and Networking, 2001.

[11] W. Rabiner Heinzelman et al., "Energy-Efficient Communication Protocols for Wireless Microsensor Networks," in the Proceedings of Hawaii International Conference on System Sciences (HICSS '00), January 2000.

[12] C. Intanagonwiwat, R. Govindan and D. Estrin, "Directed diffusion: A scalable and robust communication paradigm for sensor networks", in the Proceedings of the $6^{\text {th }}$ Annual ACM/IEEE MobiCom'00, Boston, MA, August 2000.

[13] M. Younis et al. "Energy-Aware management in Cluster-Based Sensor Networks," Computer Networks, Vol. 43, No. 5, pp. 649-668, December 2003.

[14] J.B. Andresen et al., "Propagation Measurements and Models for Wireless Communications Channels," IEEE Communications Magazine, Vol. 33, No. 1, January 1995.

[15] A. Chandrakasan et al., "Power aware wireless microsensor systems," Keynote Paper ESSCIRC, Italy 2002.

[16] F. Bai et al., "The IMPORTANT Framework for Analyzing the Impact of Mobility on Performance of Routing for Ad Hoc Networks", AdHoc Networks, Vol. 1:4, November 2000. 